\title{
Further evidence of the clinical and genetic heterogeneity of recessive transgressive PPK in the Mediterranean region
}

\author{
Cherine Charfeddine $\cdot$ Mourad Mokni • \\ Selma Kassar · Hela Zribi · Chiraz Bouchlaka • \\ Samir Boubaker · Ahmed Rebai · Amel Ben Osman · \\ Sonia Abdelhak
}

Received: 6 January 2006/ Accepted: 17 April 2006/Published online: 25 July 2006

(C) The Japan Society of Human Genetics and Springer-Verlag 2006

\begin{abstract}
Transgressive palmoplantar keratoderma (PPK) is the phenotypic hallmark of Mal de Meleda (MDM, MIM 24300). It is characterized by erythema and hyperkeratosis that extend to the dorsal face of the hands and feet. The disease is distributed worldwide and includes the Mediterranean population. The gene responsible for MDM, ARS (component $B$ ) mapped on chromosome 8qter, encodes for the SLURP-1 protein (Ly-6/uPAR related protein-1). A variety of mutations within the $A R S$ gene have been shown to underlie MDM in different populations. Genetic heterogeneity of MDM is suspected. We have recently shown that three different homozygous mutations (82delT, C77R, C99Y) were responsible for MDM in 17 patients from Northern Tunisia belonging to eight unrelated
\end{abstract}

C. Charfeddine $\cdot$ C. Bouchlaka $\cdot$ S. Abdelhak $(\bowtie)$

"Molecular Investigation of Genetic Orphan Diseases"

Research Unit (MIGOD), Institut Pasteur de Tunis, BP 74,

13 Place Pasteur, 1002 Tunis, Belvédère, Tunisia

e-mail: sonia.abdelhak@pasteur.rns.tn

M. Mokni $\cdot$ H. Zribi

Service de Dermatologie, Hôpital de la Rabta de Tunis,

Tunis, Tunisia

M. Mokni · S. Kassar · H. Zribi · A. Ben Osman

"Study of Hereditary Keratinization Disorders"

Research Unit, Hôpital La Rabta de Tunis, Tunis, Tunisia

S. Kassar $\cdot$ S. Boubaker

Service d'Anatomo-pathologie, Institut Pasteur de Tunis,

Tunis, Tunisia

A. Rebai

Service de Bioinformatique,

Centre de Biotechnologie de Sfax, Sfax, Tunisia consanguineous families. We report here a Tunisian family with three siblings presenting with recessive transgressive PPK closely resembling the MDM phenotype that excludes linkage to the $A R S$ gene.

Keywords Mal de Meleda Palmoplantar keratoderma · ARS (component B) ·

Genetic exclusion $\cdot$ Linkage analysis

\section{Introduction}

Mal de Meleda (MDM, MIM 248300), also referred to as "keratosis palmoplantaris transgrediens", is a rare inherited skin disorder classified among recessive transgressive forms of palmoplantar keratoderma (PPK). Symptoms of the disease usually appear in early infancy and are typically characterized by erythema and hyperkeratosis of the palms and soles, with sharp demarcation, that progress with age (known as progrediens) and extend to the dorsal aspects of the hands and feet (known as transgrediens) (Hovorka and Ehlers 1897; Schnyder et al. 1969). The palmoplantar hyperkeratosis is usually yellowish accompanied by hyperhidrosis, maceration, fetid odour, and painful fissures. Some commonly associated findings include nail abnormalities, keratotic plaques over the joints, perioral erythema, brachydactyly, and pseudoainhum (Bergman et al. 1993). No other organ is involved in the pathologic process. Histopathologically, hyperkeratosis, acanthosis, and foci of parakeratosis are seen (Frenk et al. 1996). Different clinical presentations of the disease have been described; these depend on the ethnic background and geographic origins of the patients (Eckl et al. 2003). Although MDM is relatively 
rare in the general population, it occurs with a relatively high frequency in some communities (Patel et al. 2001; Bakija-Konsuo et al. 2002), particularly in the Mediterranean area and the Middle East, where endogamous marriages are culturally favoured (Zahaf et al. 1987; Lestringant et al. 1997; Bouadjar et al. 2000).

MDM is inherited as an autosomal recessive trait and has been mapped to a single genetic locus on chromosome $8 \mathrm{q} 24.3$ by homozygosity mapping in several extended families (Bouadjar et al. 2000; Fisher et al. 2001; Marrakchi et al. 2003). Mutations within the $A R S$ (component $B$ ) gene (ARS) MDM have been shown to be responsible for MDM. This gene encodes a secreted Ly6/uPAR (lymphocyte antigen 6/urokinase-type plasminogen activator receptor)-related protein-1 (SLURP-1) (Fisher et al. 2001) which belongs to the Ly6/uPAR superfamily of receptor and secreted proteins (Adermann et al. 1999). SLURP-1 may modulate the intracellular calcium content of keratinocytes, thus acting as a signal in the normal growth and development of palmoplantar keratinocytes (Chimienti et al. 2003).

We have previously confirmed linkage to the $A R S$ gene in eight unrelated consanguineous Tunisian families with MDM. Genotyping of these MDM families with three microsatellite markers flanking the $A R S$ gene led to the detection of three different haplotypes, which were associated with three homozygous mutations (82delT, C77R, and C99Y) (Charfeddine et al. 2003). One of the three identified haplotypes was, furthermore, identical with an ancestral haplotype previously observed in families from Algeria and Croatia, suggesting a founder effect (Marrakchi et al. 2003; Charfeddine et al. 2003).

A genetic heterogeneity has recently been suggested for MDM (Lestringant et al. 2001; Van Steensel et al. 2002). Lestringant et al. (2001) reported five patients belonging to three consanguineous families from the United Arab Emirates who presented with an autosomal recessive transgressive PPK that closely resembles the MDM phenotype. Linkage analysis showed that the gene responsible for this phenotype is not linked either to the MDM interval or to a number of further candidate regions for PPK. Van Steensel et al. (2002) reported a Dutch MDM patient for whom no mutation of the $A R S$ gene could be found.

We report here a consanguineous Tunisian family with three siblings presenting with progredient and transgressive PPK with classical findings of MDM that did not carry a mutation in the $A R S$ gene and exclude linkage to the MDM interval.

\section{Patients and methods}

We studied a large consanguineous family from a city in central Tunisia with three patients and four unaffected family members. All available family members underwent dermatological examination. Medical history was recorded, dermatologic examination was performed by at least two dermatologists, and histopathological evaluation of skin biopsy was conducted.

Blood samples were collected from each participant family member after informed consent. DNA extraction from peripheral blood leucocytes was performed by standard procedures. Genotype and haplotype analysis were performed using the polymorphic microsatellite markers CNG003, D8S1751, and D8S1836 from centromere to telomere. Microsatellite markers span a $1 \mathrm{cM}$ interval that overlaps the MDM interval as defined by Fisher et al. (2001). Linkage analysis was performed by using the computer program Genehunther v2.1 (Kruglyak et al. 1996), assuming autosomal recessive inheritance, complete penetrance, a disease frequency of 1 per 100,000 population, and equal allele frequencies for the markers. Two-point and multipoint parametric LOD scores were calculated and the haplotypes of all the pedigree members were inferred using Genehunther. An LOD score less than or equal to -2 was regarded as significant against linkage. Mutation screening was performed by direct sequencing for affected and nonaffected individuals using intronic oligonucleotide primers (Charfeddine et al. 2003).

\section{Results}

Clinical data

Clinical characteristics of the three patients are summarized in Table 1. For patients V-2 and V-3, twin brothers 36 years old, and V-8, their sister, 23 years old, the disease appeared in early infancy and was characterized by erythema on the palms and soles, rapidly followed by a diffuse yellowish hyperkeratosis that progressed with age. The patients presented to the dermatology department for evaluation of complaints of severe diffuse yellowish, transgressive, and cracked erythrodermic PPK (Fig. 1). On the last examination the probands exhibited a thicker, irregular, rough, and scaly PPK. The transgressiveness of keratoderma included the elbows, knees, and the ulnar side of the forearms. Palmoplantar keratoderma was outlined by erythema. In all cases the sides of the feet and hands were covered by circumscribed malodor pachyderma 
Table 1 Clinical characteristics of patients of family JL

\begin{tabular}{llll}
\hline Patients' characteristics & V-2 & V-3 & V-8 \\
\hline Age (years) & 36 & 36 & 23 \\
Sex & $\mathrm{M}$ & $\mathrm{M}$ & $\mathrm{F}$ \\
Diffuse hyperkeratosis & ++ & + & ++ \\
Characteristics of transgressiveness & Dorsa of hands and feet & Dorsa of hands and feet & Glove and stocking \\
Palmoplantar hyperhidrosis & ++ & ++ & ++ \\
Malodor pachyderma & +++ & +++ & ++ \\
Nails & & ++ & ++ \\
Hyperconvexity & ++ & + Hands, feet & ++ Feet \\
Dystrophy & + Hands, feet & + & + \\
Pachyonychia & - & Elbows, knees & Hands, feet \\
Hyperkeratotic plaques & Elbows, knees & - & Hands \\
Contractures of fingers & Hands, feet & - \\
Pseudoainhum & Second, fifth fingers of both hands & + & + \\
Perioral erythema & + & - & + \\
Angular cheilitis & - & + & + \\
High arche palate & + & & + \\
\hline
\end{tabular}

+ , patient positive for characteristic; ++ , pronounced effect; - , characteristic not observed

more pronounced in patients V-2 and V-3. Hyperconvexity of the nails and conical distal phalanges were consistent features of the disease in the three patients.
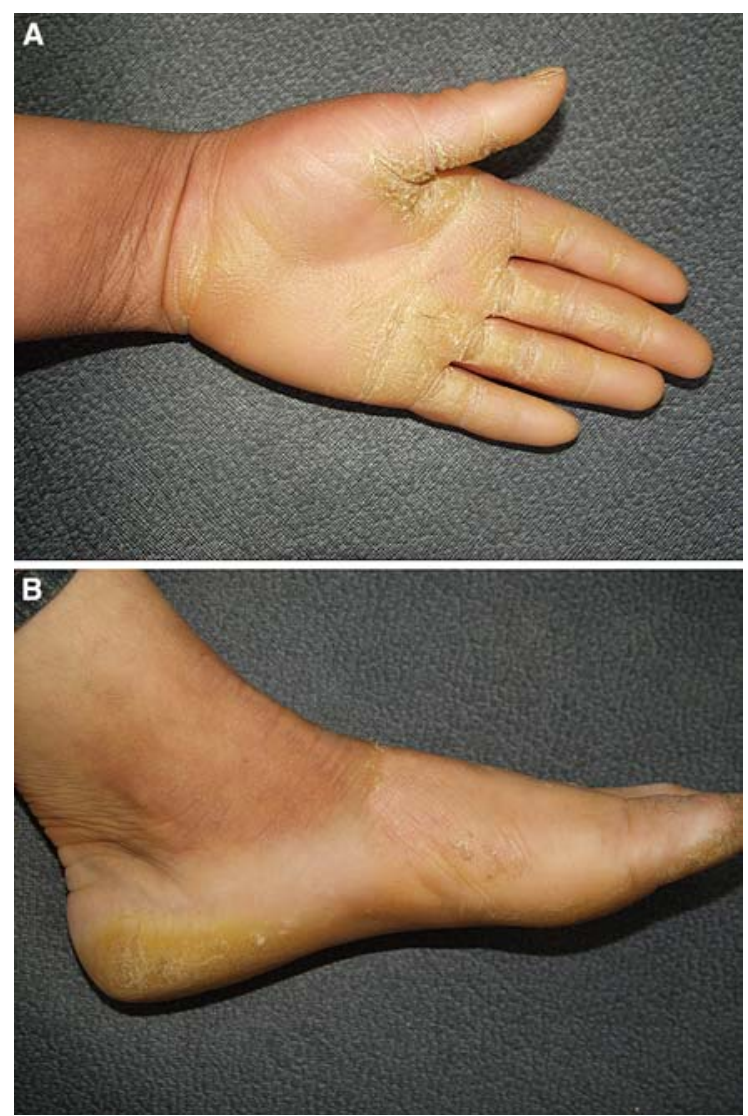

Fig. 1 A Diffuse yellowish cracked palmar keratoderma with transgrediens hyperkeratosis in patient V-8. B Diffuse yellowish plantar keratoderma with well demarcated transgrediens erythema in patient $\mathrm{V}-8$
Hyperkeratotic plaques were present on the knees and elbows of all affected family members. A pseudoainhum resulting from constricting fibrous bands of the digits was noted on the fingers of both hands of patient V-2. Perioral erythema and a high arche palate were seen in the three cases. An angular cheilitis was found in patient V-8 only. Except for these symptoms, no other physical finding was observed. Although affected siblings of the reported family had similar clinical signs, variability in presenting facultative features of the disease with regard to the nail abnormalities and pseudoainhum and perioral involvement was noted among the patients (Table 1).

None of the parents or ancestors were affected or reported affected. The genealogic tree of this family is consistent with autosomal recessive inheritance of the disorder.

Histopathological findings

After informed consent, skin biopsies were taken from the margins of the extending lesions of the wrists of the three patients. Histological examination revealed hyperkeratosis with areas of parakeratosis, hypergranulosis, acanthosis, and moderate perivascular inflammatory infiltration.

Mutation analysis and exclusion of linkage to the $A R S$ gene

On the basis of clinical features strongly suggesting the MDM phenotype and because of the relatively small size of $A R S$ gene, molecular investigation was initiated by screening one of the three affected individuals 
(patient V-2) for mutations previously reported among Tunisian MDM families. None of the previously reported mutations or other mutations was observed in exons 2 and 3 of patient V-2. Screening for mutations in exon 1 of the ARS gene also failed to reveal sequence alteration. This result suggested the patient could be mutated in the unexplored regulatory region of the ARS gene or excluded linkage to this gene. Because the family was potentially informative for linkage analysis, all available family members were genotyped using microsatellite markers overlapping the $A R S$ gene.

Haplotype analysis showed the three affected siblings were heterozygous for the two closest markers to the $A R S$ gene (Fig. 2). This is not consistent with the notion that in rare recessive inherited diseases affected children from consanguineous families are expected to be homozygous for the defective allele inherited from a common ancestor and for the genetic interval encompassing the causative gene (Lander and Botstein 1987). Affected individuals V-2 and V-3 did not, furthermore, share a common disease haplotype with their affected sister V-8, and this haplotype was shared by their clinically unaffected brother V-5. Patient V-8 also had the same disease haplotype as her healthy sister V-6. Haplotype analysis revealed several recombinations between the tested markers and the MDM locus within this family.

Multipoint parametric LOD scores were calculated for the JL family between the $A R S$ gene and the markers overlapping the $A R S$ region. Multipoint

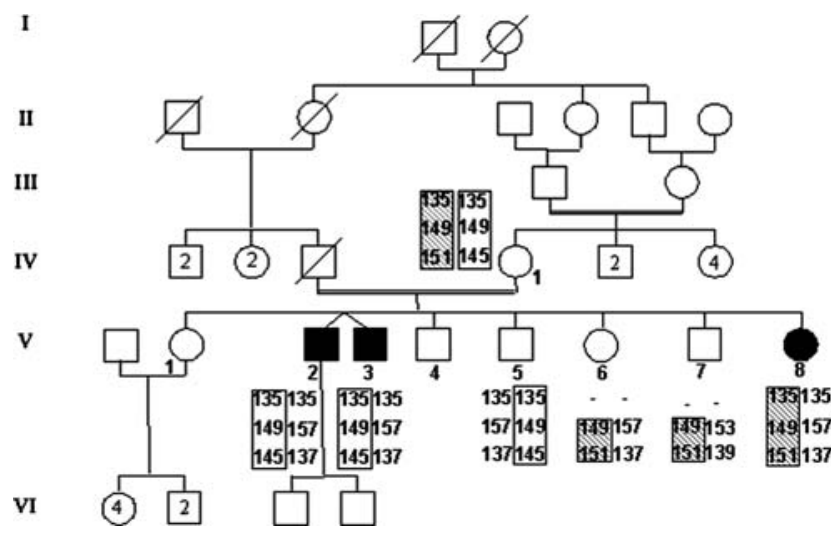

Fig. 2 Pedigree and haplotype analysis of family JL. The affected individuals are the offspring of a consanguineous marriage. Markers CNG003, D8S1751, and D8S1836 are listed from the centromere to the telomere (top to bottom). Haplotypes for markers overlapping the $A R S$ gene were derived from genotyping data and confirmed using Genehunter. The allele size of each marker is given in the base pair. The CNG003 marker was not informative for this family. Haplotype analysis revealed exclusion of linkage to the MDM interval by lack of homozygosity and several recombinations between tested markers and the $A R S$ gene analysis clearly ruled out linkage of the studied family to the MDM interval (Table 2). Two-point linkage analysis using the LINKAGE program (Lathrop and Lalouel 1984) also confirms exclusion of the region around marker D8S1836 (LOD=-2.41 at $\theta=0.001$ ).

\section{Discussion}

In this paper we report patients with early-onset, transgressive, and progrediens keratoderma associated with nail abnormalities, pseudoainhum, and perioral involvement. Taking into account common clinical features of these patients and the recessive mode of inheritance of this transgressive PPK, MDM was diagnosed. Genetic analysis excluded linkage to the $A R S$ gene. Transgressive PPK is observed in other autosomal recessive PPKs with clinical properties similar to those of MDM, for example Naxos disease (ND, MIM 601214) and Papillon-Lefèvre syndrome (PLS, MIM 248300). These can be distinguished on the basis of associated findings, i.e. cardiac abnormalities for ND and periodontopathia for PLS. Another, less severe, autosomal recessive palmoplantar erythrokeratoderma of the Gamborg Nielsen type, also known as PPK Norbotton recessive type (MIM 244850), has been reported as a possible subtle variant of MDM with no hyperkeratosis and lack of anomalies associated with MDM (Gamborg Nielsen 1985). Most other known diffuse PPK are, furthermore, inherited as an autosomal dominant trait; this was not so for the family reported here. Autosomal dominant diffuse epidermolytic PPK of Vorner (MIM 144200) and the nonepidermolytic form (Thost-Unna type) (MIM 144200) share similar clinical features but do not progress to the glove-and-socking distribution observed in MDM patients. Dermatological findings for our patients were cracked, not waxy yellow, keratoderma; these differ slightly from those of classical MDM . On the basis of differential diagnosis, the phenotype described for this family is probably closer to classical findings of MDM than to any other transgressive PPK.

Table 2 Multipoint parametric LOD scores between the $A R S$ gene and the three closest markers for the JL family

\begin{tabular}{lc}
\hline Markers & LOD scores \\
\hline CNG003 & -1.810686 \\
D8S1751 & -2.420535 \\
D8S1836 & -4.155805 \\
\hline
\end{tabular}

For the D8S1751 and D8S1836 markers closely linked to the $A R S$ gene, the JL family had a LOD score below -2 , which excludes involvement of the corresponding gene as the cause of the MDM phenotype in the three affected individuals studied 
Facultative clinical signs typical of MDM are also observed among our patients with intrafamilial variability. Clinical features observed in the patients examined also overlap those reported by Lestringant et al. (2001) for autosomal recessive transgressive PPK in patients in the United Arab Emirates.

In conclusion, this family is probably suffering from a new autosomal recessive transgressive and progrediens PPK similar to MDM, thus providing further evidence of the clinical and genetic heterogeneity of the MDM phenotype. A genome scan of this family is in progress; this will enable identification of the gene involved in this MDM-like phenotype.

Acknowledgments We wish to thank the patients and their family members. This work was supported by the Tunisian Ministry of Scientific and Technological Research and Development of Competencies (Research Unit on "Molecular Investigation of Genetic Orphan Disorders" UR 26/04 and Research Unit on "Study of Hereditary Keratinization Disorders" UR 24/ 04).

\section{References}

Adermann K, Wattler F, Wattler S, Heine G, Meyer M, Forssmann WG, Nehls M (1999) Structural and phylogenetic characterization of human SLURP-1, the first secreted mammalian member of the Ly-6/uPAR protein superfamily. Protein Sci 8:810-819

Bakija-Konsuo A, Basta-Juzbasic A, Rudan I, Situm M, Nardelli-Kovacic M, Levanat S, Fischer J, Hohl D, Loncaric D, Seiwert S, Campbell H (2002) Mal de Meleda: genetic haplotype analysis and clinicopathological findings in cases originating from the island of Mljet (Meleda), Croatia. Dermatology 205:32-39

Bergman R, Bitterman-Deutsch O, Fartasch M, Gershoni-Baruch R, Friedman-Birnbaum R (1993) Mal de Meleda keratoderma with pseudoainhum. Br J Dermatol 128:207-212

Bouadjar B, Benmazouzia S, Prud'homme JF, Cure S, Fischer J (2000) Clinical and genetic studies of 3 large, consanguineous, Algerian families with Mal de Meleda. Arch Dermatol 136:1247-1252

Charfeddine C, Mokni M, Ben Mousli R, Elkares R, Bouchlaka C, Boubaker S, Ghedamsi S, Baccouche D, Ben Osman A, Dellagi K, Abdelhak S (2003) A novel missense mutation in the gene encoding SLURP-1 in patients with Mal de Meleda from northern Tunisia. Br J Dermatol 149:1108-1115

Chimienti F, Hogg RC, Plantard L, Lehmann C, Brakch N, Fischer J, Huber M, Bertrand D, Hohl D (2003) Identification of SLURP-1 as an epidermal neuromodulator explains the clinical phenotype of Mal de Meleda. Hum Mol Genet 12:3017-3024
Eckl KM, Stevens HP, Lestringant GG, Westenberger-Treumann M, Traupe H, Hinz B, Frossard PM, Stadler R, Leigh IM, Nürnberg P, Reis A, Hennies HC (2003) Mal de Meleda (MDM) caused by mutations in the gene for SLURP-1 in patients from Germany, Turkey, Palestine, and the United Arab Emirates. Hum Genet 112:50-56

Fischer J, Bouadjar B, Heilig R, Huber M, Lefevre C, Jobard F, Macari F, Bakija-Konsuo A, Ait-Belkacem F, Weissenbach J, Lathrop M, Hohl D, Prud'homme JF (2001) Mutations in the gene encoding SLURP-1 in Mal de Meleda. Hum Mol Genet 10:875-880

Frenk E, Guggisberg D, Mevorah B, Hohl D (1996) Meleda disease: report of two cases investigated by electron microscopy. Dermatology 193:358-361

Gamborg Nielsen P (1985) Two different clinical and genetic forms of hereditary palmoplantar keratoderma in the northern most country of Sweden. Clin Genet 28:361-366

Hovorka O, Ehlers E (1897) Mal de Meleda. Arch Derm Syph (Berlin) 40:251-256

Kruglyak L, Daly MJ, Reeve-Daly MP, Lander ES (1996) Parametric and nonparametric linkage analysis: a unified multipoint approach. Am J Hum Genet 58:1347-1363

Lander ES, Botstein D (1987) Homozygosity mapping: a way to map human recessive traits with the DNA of inbred children. Science 42:1567-1570

Lathrop GM, Lalouel JM (1984) Easy calculations of LOD score and genetic risks on small computers. Am J Hum Genet 36:460-465

Lestringant GG, Frossard PM, Adeghate E, Qayed KI (1997) Mal de Meleda: a report of four cases from the United Arab Emirates. Pediatr Dermatol 14:186-191

Lestringant GG, Frossard PM, Eckl KM, Reis A, Hennies HC (2001) Genetic and clinical heterogeneity in transgressive palmoplantar keratoderma. J Invest Dermatol 116:825-827

Marrakchi S, Audebert S, Bouadjar B, Has C, Lefèvre C, Munro C, Cure S, Jobard F, Morlot S, Hohl D, Prud'homme JF, Zahaf A, Turki H, Fischer J (2003) Novel mutations in the gene encoding secreted lymphocyte antigen-6/urokinasetype plasminogen activator receptor-related protein-1 (SLURP-1) and description of five ancestral haplotypes in patients with Mal de Meleda. J Invest Dermatol 120:351-355

Patel H, Nardelli M, Fenn T, Houlston R, Coonar A, Patton MA, Crosby AH (2001) Homozygosity at chromosome 8qter in individuals affected by mal de Meleda (Meleda disease) originating from the island of Meleda. $\mathrm{Br} \mathrm{J}$ Dermatol 144:731-734

Schnyder UW, Franceschetti AT, Ceszarovic B, Segedin J (1969) Autochthonous Meleda disease. Ann Dermatol Syphiligr 96:517-530

Van Steensel MA, Van Geel MV, Steijlen PM (2002) Mal de Meleda without mutation in the $A R S$ coding sequence. Eur J Dermatol 12:129-132

Zahaf A, Charfi C, Baklouti A (1987) Kératodermie palmoplantaire type Meleda à propos de dix neufs cas. Sem Hôp Paris 63:1043-1046 